\title{
Postoperative ileus: A systematic pathway for radical cystectomy candidates?
}

\author{
Gautier Marcq, MD, MSC ${ }^{1,2,3}$; Wassim Kassouf, MD, CM, FRCSC ${ }^{1}$
}

'Division of Urology, McGill University Health Centre, McGill University, Montreal, QC, Canada; ${ }^{2}$ Univ. Lille, CNRS, Inserm, CHU Lille, Institut Pasteur de Lille, Lille, France; ${ }^{3} U$ rology department, Claude Huriez Hospital, Lille, France

Cite as: Marcq G, Kassouf W. Postoperative ileus: A systematic pathway for radical cystectomy candidates? Can Urol Assoc J 2021;15(2):40-1. http://dx.doi.org/10.5489/cuaj.7134

See related article on page 33

I $\mathrm{n}$ this issue of CUAJ, we read with great interest the study entitled, "Defining postoperative ileus and associated risk factors in patients undergoing radical cystectomy with an Enhanced Recovery After Surgery (ERAS) program" by Forbes et al. ${ }^{1}$ The authors reported a retrospective study to evaluate the rate and the associated risk factors of postoperative ileus (POI) after radical cystectomy (RC) in a high-volume center with use of ERAS protocol.

Gastrointestinal $(\mathrm{Gl})$ recovery is a cornerstone for patient management post-RC. POI remains the primary factor that leads to prolonged hospital length of stay (LOS) and increased postoperative complications and costs. ${ }^{2,3}$ ERAS protocols are now widely accepted for the perioperative management of RC; ${ }^{4}$ however, it is not clear if ERAS helps decrease POI in patients undergoing RC. A large retrospective study $(n=205)$ reported significantly lower POI rates when using ERAS protocol $(7.3 \%$ vs, $22.2 \%, p=0.003) .{ }^{5}$ In contrast, one randomized control trial (RCT) of 101 patients comparing ERAS vs. non-ERAS has shown significantly less wound healing disorders $(p=0.006)$, less fever $(p=0.004)$, and less thrombosis $(p=0.027)$ in the postoperative period, but failed to show a lower incidence of POI in patients managed with an ERAS protocol. ${ }^{6}$ Even with ERAS protocol in the current study, POI remains highly prevalent, as approximately $30 \%$ of patients developed POI according to the definition used by Forbes et al. Furthermore, none of the studied parameters were predictive of POI in their multivariate analysis.

In the initial period of ERAS development, the use of minimally invasive surgery (MIS) was recommended as an important part of the ERAS elements. ${ }^{4}$ In the RAZOR trial, a large, multicenter RCT ( $n=350)$ comparing patients managed with robot-assisted RC (RARC) vs. open RC, the rate of ileus was similar between the two approaches $(22 \%$ vs. $20 \%$, respectively). ${ }^{7}$ Bochner et al also reported on another
RCT ( $n=118) .{ }^{8}$ Unfortunately, the POI rates in both groups were not described, but the rate of high-grade complication (Clavien 3-5) was similar in both groups at around 20\% $(p=0.90)$. In the CORAL trial, a small, single-center RCT $(n=60)$, patients were randomized between RARC, laparoscopic RC, and open RC. The rate of ileus was higher in the open RC arm. ${ }^{9}$ Importantly, in all reported randomized trials, the patients in the RARC arm underwent extracorporeal urinary diversions. Whether performing RARC with intracorporeal diversion decreases POI and/or complications requires further evaluation in a randomized trial.

Several non surgical-related therapies have been reported to potentially improve bowel recovery time, such as the use of early mobilization, early feeding, gastrointestinal stimulation with metoclopramide, and chewing gum. ${ }^{4,10}$ In 2013, the U.S. Food and Drug Administration (FDA) approved the use of alvimopan, a peripherally acting $\mu$-opioid receptor antagonist, based on several RCTs in patients with abdominal surgeries, including bowel resection. For RC, a large, placebo-controlled, multicenter RCT (21 centers, 277 patients included) investigated the role of oral alvimopan. ${ }^{11}$ On the day of surgery, patients were randomized to receive single-dose $(12 \mathrm{mg}$ ) oral alvimopan (Entereg, Cubist Pharmaceuticals, Inc.) or matching placebo between 30 minutes and five hours before surgery start, then twice daily until hospital discharge or a maximum of seven days. The endpoint was defined as time to upper (first tolerance of solid food) and lower (first bowel movement) Gl recovery. Authors reported quicker time to the endpoint with the use of alvimopan compared to placebo (5.5 vs. 6.8 days; $\mathrm{p}<0.0001$ ), as well as a shorter mean LOS (7.4 vs. 10.1 days; $\mathrm{p}=0.0051$ ) and fewer episodes of POI-related morbidity $(8.4 \%$ vs. $29.1 \% ; p<0.001)$. Of note, this study was performed on patients who did not receive epidural analgesia.

Unfortunately, alvimopan is not offered to patients in Canada, as this drug has not been Health Canadaapproved..$^{12}$ As per Health Canada, a notice of non-compliance $(\mathrm{NON})$ was issued due to deficiencies in the chemistry and manufacturing component of alvimopan. The sponsor chose to cancel their submission after receiving the NON. 
To the best of our knowledge, no other therapy has shown such results to improve $\mathrm{POI}$ after $\mathrm{RC}$, thus reconsidering drug approval would be timely for our patients. In the meantime, implementation of non-opioid protocols have been advocated in some centers for patients undergoing $\mathrm{RC}$ to minimize $\mathrm{POI}$ rates. ${ }^{13}$

As explained by Forbes et al in this issue, the rate of $\mathrm{POI}$ can drastically vary upon the definition used. In their study, $\mathrm{POI}$ rates ranged from $1-51 \%$ according to several reported POI definitions. This notion has an important impact when comparing data across studies and outlines the unmet need for a standardized definition of POI when evaluating RC outcomes. In 2018, the Perioperative Quality Initiative from the American Society for Enhanced Recovery published a consensus statement on postoperative Gl dysfunction within an enhanced recovery pathway for elective colorectal surgery. ${ }^{14}$ Their first recommendation was: "Consider foregoing the traditional definition of ileus for the Intake, Feeling nauseated, Emesis, physical Exam, and Duration of symptoms (I-FEED) scoring system - a more functional definition of postoperative Gl dysfunction (POGD) that takes into account the wide spectrum of signs, symptoms, and associated clinical implications." The I-FEED scoring system allows an objective definition of impaired postoperative Gl function. These notions can be easily implemented into clinical trial design, rendering the feasibility of cross-trial comparisons in the future.

In conclusion, even with the implementation of ERAS protocols, $\mathrm{POI}$ remains a common problem after RC. There is an unmet need to further improve postoperative care for this commonly frail population through well-designed clinical trials.

Competing interests: Dr. Kassouf has received grants/honoraria from Amgen, Astellas, and Janssen. Dr. Marcq reports no competing personal or financial interests related to this work.

\section{References}

1. Forbes $C M$, Chehroudi AC, Mannas M, et al. Defining postoperative ileus and associated risk factors in patients undergoing radical cystectomy with an Enhanced Recovery After Surgery (ERAS) program. Can Urol Assoc J 2021;15(2):33-9. http://dx.doi.org/10.5489/cuaj.6546

2. Chang SS, Baumgartner RG, Wells $\mathrm{N}$, et al. Causes of increased hospital stay after radical cystectomy in a clinical pathway setting. J Urol 2002;167:208-11. https://doi.org/10.1016/S0022-5347(05)65414-6

3. Doorly MG, Senagore AJ. Pathogenesis and clinical and economic consequences of postoperative ileus. Surg Clin North Am 2012;92:259-72. https://doi.org/10.1016/i.suc.2012.01.010

4. Cerantola Y, Valerio M, Persson B, et al. Guidelines for perioperative care after radical cystectomy for bladder cancer: Enhanced Recovery After Surgery (ERAS ${ }^{\circledR}$ ) society recommendations. Clin Nutr 2013;32:879-87. https://doi.org/10.1016/i.clnu.2013.09.014

5. Xu W, Daneshmand S, Bazargani ST, et al. Postoperative pain management after radical cystectomy: Comparing traditional vs. Enhanced Recovery Protocol Pathway. J Urol 2015;194:1209-13. https:// doi.org/10.1016/i.juro.2015.05.083

6. Karl A, Buchner A, Becker A, et al. A new concept for early recovery after surgery for patients undergoing radical cystectomy for bladder cancer: Results of a prospective randomized study. J Urol 2014;191:335-40. https://doi.org/10.1016/i.juro.2013.08.019

7. Parekh DJ, Reis IM, Castle EP, et al. Robot-assisted radical cystectomy vs. open radical cystectomy in patients with bladder cancer (RAZOR): An open-label, randomized, phase 3, non-inferiority trial. Lancet Lond Engl 2018;391:2525-36.

8. Bochner BH, Sjoberg DD, Laudone VP. Memorial Sloan Kettering Cancer Center Bladder Cancer Surgical Trials Group. A randomized trial of robot-assisted laparoscopic radical cystectomy. N Engl J Med 2014;371:389-90. https://doi.org/10.1056/NEJMc1 405213

9. Khan MS, Gan C, Ahmed K, et al. A Single-center, early-phase randomized controlled three-arm trial of open, robotic, and laparoscopic radical cystectomy (CORAL). Eur Urol 2016;69:613-21. https://doi. org/10.1016/i.eururo.2015.07.038

10. Kouba EJ, Wallen EM, Pruthi RS. Gum chewing stimulates bowel motility in patients undergoing radical cystectomy with urinary diversion. Urology 2007;70:1053-6. https://doi.org/10.1016/i. urology.2007.07.048

11. Lee CT, Chang SS, Kamat AM, et al. Avvimopan accelerates gastrointestinal recovery after radical cystectomy: A multicenter, randomized, placebo-controlled trial. Eur Urol 2014;66:265-72. https://doi. org/10.1016/i.eururo.2014.02.036

12. Government of Canada HC. Search Page - Drug and Health Product Register [Internet]. 2014. Available at: https://hpr-rps.hres.ca/reg-content/regulatory-decision-summary-detailTwo.php?lang=en\&linkID=R DS00231 \&wbdisable=true. Accessed December 13, 2020

13. Audenet $F$, Attalla $\mathrm{K}$, Giordano $\mathrm{M}$, et al. Prospective implementation of a nonopioid protocol for patients undergoing robot-assisted radical cystectomy with extracorporeal urinary diversion. Urol Oncol 2019;37:300.e17-23. https://doi.org/10.1016/i.urolonc.2019.02.002

14. Hedrick TL, McEvoy MD, Mythen MMG, et al. American Society for Enhanced Recovery and Perioperative Quality Initiative joint consensus statement on postoperative gastrointestinal dysfunction within an enhanced recovery pathway for elective colorectal surgery. Anesth Analg 2018;126:1896-907.

Correspondence: Dr. Wassim Kassouf, Department of Urology, McGill University Health Centre, Montreal, QC, Canada; wassim.kassouf@muhc.mcgill.ca 